\title{
La motivación en el deporte adaptado
}

\author{
Miguel Ángel Torralba ${ }^{1 *}$, Marcelo Braz y Maria José Rubio \\ ${ }^{1}$ Universidad de Barcelona
}

(Recibido 12 abril 2014; Aceptado 27 abril 2014)

RESUMEN: El presente estudio examina la motivación hacia la práctica deportiva de personas con discapacidad que forman parte del deporte federado. La muestra está compuesta por 134 deportistas de ambos géneros y diferente discapacidad. Como instrumento se ha utilizado el Cuestionario "Participation Motivation Inventory", de Gill, Gross y Huddleston, adaptado al deporte Paralímpico y que describe las principales razones que incitan a la actividad deportiva. En los resultados no se han encontrado diferencia significativa entre hombres y mujeres, ni entre ciegos - discapacitados visuales y discapacitados físico motriz. Sobre la motivación de la práctica deportiva, cabe destacar la importancia dada a los factores de condición física y salud, tales como practicar deporte, mejorar el nivel, competir, sentirse bien o divertirse, muy por encima de por ser popular, por influencia de los entrenadores o satisfacer a los padres.

Palabras clave: deporte adaptado, motivación deportiva, discapacidad.

\section{Motivation in adapted sport}

\begin{abstract}
This study examines the motivation for practice of sport of people with disabilities that form part to a federated sport.The sample was composed of 134 athletes of both genders and different disabilities.The "Participation Motivation Inventory Questionnaire" by Gill, Gross and Huddleston was used. The instrument was adapted to Paralympic sport and describes the main reasons that encourage the sports activity practice. The results haven't found significant difference between men's and women's or between blind - visually impaired physical and motor disabilities. About the motivation of the practice of sport, worth highlighting the importance given to factors of fitness and health, like sport practice, improve the level, to compete, feel good and have fun, well above being popular, influenced by coaches or satisfy to parents.
\end{abstract}

Keywords: adapted sports, sports motivational, disability.

\section{INTRODUCCIÓN}

En el último lustro se ha transformado positivamente la percepción social de las personas con discapacidad. No es una evolución meramente ornamental, sino que expresa un cambio de mentalidad, una metamorfosis de la mirada. Afortunadamente ya vivimos en una sociedad donde se reconocen sus derechos inherentes y esto tiene repercusión en todos los planos: educativo, social, deportivo, laboral y afectivo. Ya no se ve la discapacidad como un mero ser de necesidades, sino 
también como un ser con posibilidades, con un potencial y una riqueza de recursos para desarrollar y cultivar.

Afortunadamente vivimos en una sociedad donde se está normalizando la situación de las personas con discapacidad y en el ámbito deportivo los Juegos Paralímpicos promueven un modelo de inclusión donde las personas pueden demostrar todas sus capacidades y contribuir a un cambio de mentalidad, una metamorfosis de la mirada.

La inclusión de las personas con discapacidad en todas las facetas de la vida en sociedad es una constante en nuestros días. En muchos países del mundo, se han aplicado nuevas leyes con el fin de ofrecer igualdad de oportunidades y plena participación en la vida social a todos los miembros de la sociedad, poniendo especial énfasis en la situación de las personas con discapacidad. Programas deportivos en el ámbito mundial, como los Juegos Paralímpicos, realizados con posterioridad a los Juegos Olímpicos y desarrollados en la misma sede, creación de Federaciones Internacionales y Nacionales de las diferentes discapacidades, etc., posibilitan esta integración.

La O.M.S. (1980) define que la discapacidad es toda restricción o ausencia de la capacidad de realizar una actividad en la forma o dentro del margen que se considera normal para el ser humano.

Cuando se habla de origen del deporte paralímpico hay que mencionar al neurólogo Ludwig Guttmann (1899-1980), padre e impulsor del deporte como forma de rehabilitación en las personas con discapacidad.

Al acabar la guerra, la sociedad europea tenía dos grandes problemas que resolver, el primero era el destrozo de ciudades y el segundo era la inclusión de las personas con discapacidad en la vida social. Al segundo problema se le busco una solución, el deporte. Así el neurólogo desarrolló programas de recuperación mediante el deporte. Su objetivo era ayudar a un gran número de soldados lesionados de la columna vertebral. No solo ayudaba recreativamente sino que también mejoraba la rehabilitación física y psicológica.

Los beneficios que obtenían llevaron a plantear el competir con otros hospitales de la misma nación. La celebración coincidió con los Juegos Olímpicos de Londres (1948).

En 1960 se cumplió el sueño de Guttmann y de muchos deportistas, el profesor italiano Antonio Maglio, director del Centro de Lesiones Medulares (INAIL) de Ostia, propuso que los I Juegos Internacionales de Stoke Mandeville se celebraran en Roma, coincidiendo con los Juegos Olímpicos, aunque posteriormente no siempre siguió siendo así. Las "Olimpiadas para minusválidos" no fueron aprobadas por el Comité Olímpico Internacional hasta 1984 (Comité paralímpico Español 2006).

Hasta que llegó a los ámbitos competitivos el deporte practicado por atletas con un tipo u otro de discapacidad, la homologación de los contendientes se hacía tomando en cuenta la edad, el sexo, o el peso de cada uno, o bien las desventajas que pudieran influir en la realización del deporte correspondiente. La gran variedad de discapacidades existentes en el mundo de las personas con minusva- 
lía, ninguna es igual a otra, creó una obligación adicional a los responsables de la organización deportiva y fue la ordenación en grupos de competición. Pronto se pasó del deporte lúdico a deporte de competición, realizándose las primeras clasificaciones entre los deportistas mediante un método simple, como era valorar sus secuelas patológicas, formándose grupos de parapléjicos, de amputados, de invidentes, etc. Con el tiempo, dentro de dichos grupos se fue introduciendo subgrupos, ya que fue obvio que, aunque su patología de base fuese la misma, el grado de afectación era muy dispar entre ellos.

Así, a los deportistas minusválidos con lesión medular, se les dividió según el nivel de afectación medular. A los amputados, según si eran de extremidades superiores o de sus extremidades inferiores, dobles o simples. Los invidentes, si eran totales o parciales. Con lo cual, en esta evolución histórica, vemos que las primeras valoraciones no eran más que definiciones de secuelas patológicas y sus distintos grados de afectación, sistema que, hoy por hoy, todavía utilizamos en la práctica diaria al comparar varios deportistas minusválidos.

El Comité Paralímpico Internacional y el Comité Español (2006) organizan al deporte paralímpico en cuatro grandes grupos según el grado de discapacidad: ciegos, discapacitados intelectuales, minusválidos físicos y paralíticos cerebrales.

El primer tipo de discapacidad lo forman las personas ciegas o con discapacidad visual, es decir, con alguna característica que impida una visión "normal". Esto incluye toda la gama de problemas visuales, desde disfunciones que pueden corregirse hasta la ceguera total.

Los criterios de elegibilidad de las personas con discapacidad intelectual están actualmente en revisión, aunque vienen utilizando los aceptados por la Organización Mundial de la Salud (OMS) y la Asociación Americana del Retraso Mental, y que en conjunto son tres: tener un coeficiente intelectual igual o inferior a 70, tener limitaciones en dos o más áreas de adaptación social y haber adquirido su condición antes de los 18 años.

Dentro de los deportistas discapacitados físicos se enmarcan las personas amputadas, que son personas que tienen, al menos, una articulación principal en un miembro amputado (codo, muñeca, rodilla...). También dentro de este grupo existen deportistas con lesiones medulares, y los denominados "les autres" (los otros), que son los que tienen disfunciones en el sistema locomotor. Se incluyen también personas en silla de ruedas que deben tener como mínimo un 10 por ciento de pérdida funcional en seis miembros inferiores.

Finalmente el último grupo son los deportistas con parálisis cerebral, una disfunción del movimiento producida por una lesión en una o varias zonas del cerebro que controlan y coordinan el tono muscular, los reflejos, las posturas y movimientos.

Algunos deportes están abiertos a todo tipo de discapacidades como atletismo o natación, en cambio en otros sólo participan un tipo de minusvalía. En judo, goolball, por ejemplo participan los deficientes visuales y en boccia las personas con parálisis cerebral. 
Pero el deporte adaptado, al igual que el deporte no adaptado,tiene muchas aceptaciones que van desde el deporte escolar al deporte federado de competición, pasando por el deporte salud y deporte para todos.

Nosotros en este trabajo hemos querido conocer las motivaciones de los deportistas federados que participan en el deporte adaptado. La motivación hacia el deporte ha sido tradicionalmente una de las preocupaciones principales de los estamentos deportivos, debiendo llegar a conocer cuáles son los motivos que llevan a nuestros deportistas a la práctica de este. Para contribuir a este objetivo y empleando como marco de referencia la teoría de las metas de logro, en el presente estudio se analizan los motivos de la práctica deportiva de deportistas participantes en los Juegos Paralímpicos de Londres y en competiciones deportivas nacionales de las Federaciones de Deporte Adaptado de España.

La población que practica deporte adaptado federado está distribuida en cinco federaciones: Ciegos y discapacitados visuales, Personas con discapacidad física, Personas con parálisis cerebral, Discapacitados intelectuales y Sordos.

La población que nosotros hemos estudiado ha estado compuesta por 134 deportistas de ambos géneros y diferente discapacidad ( 55 de la categoría ciegos y discapacitados visuales y 79 de la categoría físico-motriz). Se ha utilizado como instrumento el Cuestionario de Motivos de Participación en la práctica de Deporte Paralímpico, compuesto por 30 ítems, y basado en el cuestionario"Participatio nMotivationInventory", que describe las principales razones que incitan a la actividad deportiva y que ha sido exitosamente utilizado (Gill, Gross y Huddleston, 1983) y que varios autores lo han utilizado para analizar los motivos de práctica deportiva en diferentes deportes, tanto respetando la versión original en lengua inglesa (Gould, Feltz, y Weiss, 1985; Klint y Weiss, 1987; Ryckman y Hamel, 1993) como haciendo adaptaciones a diferentes idiomas: español (Durand, 1988; Balaguer y Atienza, 1994; Lázaro, Villamarin y Limonero, 1996; Cecchini, Mendez y Muñiz, 2002), chino (Wang y Wiese-Bjornstal, 1996) y otros.

El tratamiento de las personas con discapacidad ha sido estudiado en el ámbito de la actividad física y deportiva por autores como Guthrie (1999), Guthrie y Castellnuovo (2001) y Martín (1999), según refiere Gutierrez (2006). Gran parte de la literatura referente a la actividad física y deporte adaptado ve este campo como un medio para la normalización de la persona, es decir, con un fin utilitario, pero en escasas ocasiones que hagan referencia a la práctica de la actividad física como un fin en sí misma. De este modo, la mayoría de los trabajos se han limitado a un estudio descriptivo comparando las características de las personas con y sin discapacidad en el ámbito deportivo pero sin estar fundamentados en un modelo teórico para comprender la motivación del comportamiento. Nosotros analizamos las diferencias de edad, género, años de práctica deportiva y categoría (ciegos y físicos-paralíticos cerebrales), siendo los resultados agrupados en 4 factores: Condición física - Salud, Social, Autoestima y Competencia. 


\section{MÉTODO}

\section{Participantes}

La muestra estuvo compuesta por un total de 134 deportistas (104 hombres y 30 mujeres) practicantes del deporte adaptado a nivel autonómico y nacional de España, con edades comprendidas entre16 y 63 años, con un promedio de 30,37 $( \pm 8,29)$. Los participantes pertenecían a once modalidades deportivas (atletismo, baloncesto, boccia, ciclismo, futbol, halterofilia, natación, tenis, tenis de mesa, tiro olímpico y vela) divididos en dos categorías: Ciegos y discapacitados visuales, y Físicos.Los años de práctica deportiva de éstos variaron entre 1 y 39 años, con un promedio de $12,78( \pm 7,51)$.

Tabla 1. Muestra

\begin{tabular}{|c|c|c|c|c|}
\hline \multirow{2}{*}{\multicolumn{2}{|c|}{ Tipo de discapacidad }} & \multicolumn{2}{|c|}{ Género } & \multirow{2}{*}{ Total } \\
\hline & & Masculino & Femenino & \\
\hline \multicolumn{2}{|c|}{ Ciegos y Discapacitados Visuales } & 41 & 14 & 55 \\
\hline & Físicos & 63 & 16 & 79 \\
\hline & Total & 104 & 30 & 134 \\
\hline & $\%$ total & $77,6 \%$ & $22,4 \%$ & $100 \%$ \\
\hline \multirow{2}{*}{ Edad } & Min-Máx & \multicolumn{3}{|c|}{$16-63$} \\
\hline & Media (DT) & \multicolumn{3}{|c|}{$30,37( \pm 8,29)$} \\
\hline \multirow{2}{*}{$\begin{array}{l}\text { Tiempo de } \\
\text { Práctica }\end{array}$} & Min-Máx & \multicolumn{3}{|c|}{$1-39$} \\
\hline & Media (DT) & \multicolumn{3}{|c|}{$12,78( \pm 7,51)$} \\
\hline
\end{tabular}

\section{Instrumento}

El cuestionario utilizado en esta investigación es la versión en castellano del "Participation Motivation Inventory" (Gill y cols., 1983) presentada en diversas investigaciones (Durand, 1988; Balaguer y Atienza, 1994; Lázaro y cols., 1996; Cecchiniy cols., 2002). En este cuestionario los participantes han de valorar la importancia de una serie de motivos por los cuáles practican deporte adaptado. Para cada uno de los 30 ítems, los deportistas responden si creen que ese motivo es "muy importante", "algo importante" o "nada importante" y rellenan unos datos generales sobre categoría deportiva, edad, sexo y años de práctica deportiva.

La administración de los cuestionarios se llevó a cabo en el año 2012 y se pidió la colaboración de los delegados y técnicos deportivos de los diferentes equipos autonómicos y nacionales, a los que se les explicó detalladamente los 
objetivos del estudio, facilitando las orientaciones de la ejecución y relleno del cuestionario.

\section{Análisis estadístico}

El análisis de los datos se realizó con el programa estadístico SPSS 21.0 para Windows y tuvo como objetivos: a) identificar las dimensiones de la escala y su fiabilidad; b) describir las respuestas de los deportistas en cada una de las 30 razones expuestas en la escala; y c) comprobar posibles diferencias por sexo y tipo de discapacidad.

Para identificar las dimensiones de la escala se llevó a cabo un análisis factorial de los ítems con método de Componentes Principales y rotación Varimax, el cual identificó una estructura factorial de 4 componentes interpretables, que denominamos: Condición física - Salud, Social, Autoestima y Competencia. Para verificar la fiabilidad como consistencia interna del instrumento se utilizó el coeficiente de fiabilidad Alfa de Cronbach. Para la descripción de las respuestas de los deportistas se utilizaron los indicadores de tendencia central y dispersión más habituales (media y desviación típica) y los porcentajes de los diferentes niveles de la escala; y para identificar las diferencias entre grupos se aplicó la prueba de contraste de medias t de Student.

\section{RESULTADOS}

\section{Análisis factorial}

En el análisis factorial exploratorio realizado, todos los ítems (a excepción de tres) obtuvieron una carga superior a 0,40 en el factor relevante (Nunnally y Bernstein, 1995). Las cargas por ítem de cada factor se pueden ver en la tabla 2. El test de Bartlett confirmó que el análisis factorial era procedente $(p=, 000)$. A partir de la revisión del análisis de los cuatro componentes, se excluyeron tres ítems (4. "Quiero gastar energía”, 10. "Quiero aprender nuevas habilidades", 13. "Quiero relajarme") debido al bajo valor alcanzado. La varianza total explicada para los cuatro factores es de 51,3\%, siendo el factor más explicativo el de Condición fisica con un $16,59 \%$ de varianza explicada. Para el social se obtuvo un $13,80 \%$, para la autoestima un $11,60 \%$ y para la competencia un $9,03 \%$. 
Tabla 2. Análisis Factorial Exploratorio

\begin{tabular}{|c|c|c|c|c|}
\hline \multirow{2}{*}{\multicolumn{5}{|c|}{$\begin{array}{l}\text { Factores } \\
\text { Condición Física / Salud }\end{array}$}} \\
\hline & & & & \\
\hline 01. Quiero mejorar mis habilidades & 0,65 & & & \\
\hline 03. Me gusta ganar & 0,55 & & & \\
\hline 06. Quiero estar en forma & 0,68 & & & \\
\hline 15.Me gusta hacer ejercicio & 0,59 & & & \\
\hline 20. Me gusta la competición & 0,52 & & & \\
\hline 22. Me gusta practicar deporte & 0,62 & & & \\
\hline 23. Quiero mejorar mi nivel & 0,76 & & & \\
\hline 24. Quiero estar físicamente bien & 0,76 & & & \\
\hline 29. Me gusta divertirme & 0,58 & & & \\
\hline \multicolumn{5}{|l|}{ Social } \\
\hline 02. Quiero estar con mis amigos & & 0,65 & & \\
\hline 05. Me gusta viajar & & 0,44 & & \\
\hline 08. Me gusta el trabajo en equipo & & 0,74 & & \\
\hline 11.Me gusta hacer nuevos amigos & & 0,71 & & \\
\hline 16. Me gusta tener algo que hacer & & 0,51 & & \\
\hline 18. Me gusta el espíritu de equipo & & 0,77 & & \\
\hline 19. Me gusta salir de casa & & 0,66 & & \\
\hline 30. Me gusta utilizar el equipo o instalaciones & & 0,56 & & \\
\hline \multicolumn{5}{|l|}{ Auto estima } \\
\hline 09. Es por satisfacer a mis padres & & & 0,60 & \\
\hline 12. Me gusta hacer algo en lo que destaco & & & 0,53 & \\
\hline 14. Me gustan las recompensas & & & 0,52 & \\
\hline 21. Me gusta sentirme importante & & & 0,73 & \\
\hline 25. Quiero ser popular & & & 0,77 & \\
\hline 27. Por influencia de los entrenadores & & & 0,58 & \\
\hline 28. Quiero alcanzar un status-élite & & & 0,49 & \\
\hline \multicolumn{5}{|l|}{ Competencia } \\
\hline 07. Me gustan las emociones & & & & 0,69 \\
\hline 17. Me gusta la acción & & & & 0,73 \\
\hline 26. Me gustan los desafíos & & & & 0,68 \\
\hline Auto valores & 4,47 & 3,72 & 3,13 & 2,51 \\
\hline$\%$ de la varianza & 16,6 & 13,8 & 11,6 & 9,03 \\
\hline$\%$ varianza total & & & & 51,3 \\
\hline
\end{tabular}

\section{Fiabilidad}

La fiabilidad, entendida como la consistencia interna del instrumento, se obtuvo mediante el coeficiente Alfa de Cronbach, el cual arrojó diferentes valores para cada una de las dimensiones o factores identificados en el análisis factorial. Para el factor "Condición física / Salud" formado por 9 ítems el alfa fue igual a 0,851 . El factor "Social" arrojó un alfa de 0,820 en sus 8 elementos. El factor "Autoestima" obtuvo el valor alfa de 0,754 en sus 7 elementos y el factor "Com- 
petencia" alcanzó un alfa de 0,735 para sus 3 elementos. Con relación a la media y desviación típica, se puede observar que la autoestima es el factor con mayor valor $(13,79)$, pero muy próximo del factor social $(12,81)$ y de la condición física / salud $(11,13)$. Muy lejos queda el factor competencia $(4,23)$, como demuestra la Tabla 3.

Tabla 3. Análisis Descriptivo y de la Consistencia Interna de los Factores

\begin{tabular}{lcccc}
\hline Factores & M & DT & Alfa de Cronbach & N de elementos \\
\hline Condición Física / Salud & 11,13 & 2,99 & 0,851 & 9 \\
Social & 12,81 & 3,55 & 0,820 & 8 \\
Autoestima & 13,79 & 3,17 & 0,754 & 7 \\
Competencia & 4,23 & 1,41 & 0,735 & 3 \\
\hline
\end{tabular}

$\mathrm{M}$ - media; DT - desviación típica.

\section{Análisis descriptivo}

En la Tabla 4 se exponen los datos descriptivos de los ítems de la escala (Media, Desviación Típica, Número total de participantes y Porcentaje de la importancia concedida, considerando el nivel de la escala "muy importante"). Los datos están ordenados de acuerdo con el porcentaje de importancia del ítem. La media alcanzada por cada ítem también indica su nivel de importancia siendo más importante cuanto más se acerca a 1.

Podemos verificar que los ítems "Me gusta practicar deporte individual", "Quiero mejorar mi nivel", "Me gusta hacer ejercicio", "Me gusta la competición", "Quiero estar físicamente bien" y "Me gusta divertirme" están por encima del $75 \%$ de valoración, lo que indica que son elementos muy importantes para los deportistas.

Por otro lado apenas tres ítems indican valoración de importancia por debajo de $\operatorname{los} 25 \%$ ("Es por satisfacer a mis padres", "Por influencia de los entrenadores" y "Quiero ser popular"). 
Tabla 4. Análisis Descriptivo

\begin{tabular}{|c|c|c|c|c|c|}
\hline & \multicolumn{3}{|c|}{ Datos generales } & \multicolumn{2}{|c|}{$\begin{array}{c}\text { Porcentaje } \\
\text { Muy Importante }\end{array}$} \\
\hline & Media & DT & $\mathrm{N}$ total & $\%$ & $\mathrm{~N}$ \\
\hline 22. Me gusta practicar deporte individual & 1,16 & ,462 & 134 & 87,5 & 119 \\
\hline 23. Quiero mejorar mi nivel & 1,16 & 428 & 134 & 85,3 & 116 \\
\hline 15. Me gusta hacer ejercicio & 1,21 & ,460 & 134 & 81,6 & 111 \\
\hline 20. Me gusta la competición & 1,24 &, 523 & 134 & 80,1 & 109 \\
\hline 24. Quiero estar físicamente bien & 1,24 & ,478 & 134 & 77,9 & 106 \\
\hline 29. Me gusta divertirme & 1,27 &, 508 & 134 & 76,5 & 104 \\
\hline 03. Me gusta ganar & 1,31 &, 565 & 134 & 74,6 & 100 \\
\hline 06. Quiero estar en forma & 1,23 & ,474 & 134 & 74,3 & 101 \\
\hline 01. Quiero mejorar mis habilidades & 1,31 &, 538 & 134 & 73,5 & 100 \\
\hline 26. Me gustan los desafíos & 1,34 &, 563 & 134 & 69,1 & 94 \\
\hline 17. Me gusta la acción & 1,46 & ,621 & 134 & 61,0 & 83 \\
\hline 08. Me gusta el trabajo en equipo & 1,49 & ,646 & 134 & 58,8 & 80 \\
\hline 18. Me gusta el espíritu de equipo & 1,51 & ,657 & 134 & 58,1 & 79 \\
\hline 12. Me gusta hacer algo en lo que destaco & 1,52 &, 597 & 134 & 53,7 & 73 \\
\hline 10. Quiero aprender nuevas habilidades & 1,54 & ,633 & 134 & 53,0 & 71 \\
\hline 07. Me gustan las emociones & 1,42 & ,566 & 134 & 52,9 & 72 \\
\hline 11. Me gusta hacer nuevos amigos & 1,57 & 631 & 134 & 50,7 & 69 \\
\hline 30. Me gusta utilizar el equipo o instalaciones & 1,59 & ,663 & 134 & 50,0 & 68 \\
\hline 28. Quiero alcanzar un status-élite & 1,65 & ,728 & 134 & 50,0 & 68 \\
\hline 16. Me gusta tener algo que hacer & 1,60 & ,615 & 134 & 47,1 & 64 \\
\hline 02. Quiero estar con mis amigos & 1,65 & ,686 & 134 & 47,1 & 64 \\
\hline 14. Me gustan las recompensas & 1,65 & 697 & 133 & 46,3 & 63 \\
\hline 05. Me gusta viajar & 1,66 & ,684 & 133 & 46,3 & 63 \\
\hline 19. Me gusta salir de casa & 1,78 & ,708 & 134 & 38,2 & 52 \\
\hline 21. Me gusta sentirme importante & 1,87 & ,740 & 134 & 34,6 & 47 \\
\hline 04. Quiero gastar energía & 1,82 & 692 & 134 & 34,3 & 46 \\
\hline 13. Quiero relajarme & 1,93 & ,709 & 133 & 28,4 & 38 \\
\hline 09. Es por satisfacer a mis padres & 2,41 & ,759 & 133 & 17,6 & 24 \\
\hline 27. Por influencia de los entrenadores & 2,29 & ,734 & 134 & 16,2 & 22 \\
\hline 25. Quiero ser popular & 2,36 &, 732 & 132 & 15,4 & 21 \\
\hline
\end{tabular}

En la Tabla 5 se presentanlos datos descriptivos (media, desviación típica y porcentaje de cada nivel de la escala) de los ítems dentro de sus respectivos factores resultantes del análisis factorial. Como se puede observar, el motivo más valorado por los deportistas en el factor "Condición Física / Salud" es el "15. Me gusta practicar deporte" $(\mathrm{M}=1,16)$, donde $87,5 \%$ de los deportistas indican el motivo como muy importante. 
En el factor "Social" el motivo más valorado fue "08. Me gusta el trabajo en equipo" $(\mathrm{M}=1,49)$ con un $58,8 \%$ de los deportivas que dicen que el motivo es muy importante. En este factor, es posible verificar una aproximación entre las respuestas muy importante y algo importante en prácticamente todos los motivos.

En el factor "Autoestima" 53,7 \% de los deportistas indicaron que el motivo "12. Me gusta hacer algo en lo que destaco" $(M=1,51)$ es el más importante. Y, en el factor "Competencia" 69,1\% de los deportistas revelan como más importante el motivo "26. Me gustan los desafíos" $(\mathrm{M}=1,35)$.

Tabla 5. Frecuencia de las Respuestas

Muy

Algo

Nada

importante importante importante

\begin{tabular}{|c|c|c|c|c|c|c|c|c|}
\hline Factores & $\mathrm{M}$ & DT & $\mathrm{f}$ & $\%$ & $\mathrm{f}$ & $\%$ & $\mathrm{f}$ & $\%$ \\
\hline \multicolumn{9}{|l|}{ Condición Física / Salud } \\
\hline 01. Quiero mejorar mis habilidades & 1,30 & 0,54 & 100 & 73,5 & 31 & 22,8 & 5 & 3,7 \\
\hline 03. Me gusta ganar & 1,31 & 0,57 & 101 & 74,3 & 28 & 20,6 & 7 & 5,1 \\
\hline 06. Quiero estar en forma & 1,23 & 0,47 & 101 & 74,3 & 25 & 18,4 & 3 & 2,2 \\
\hline 15. Me gusta hacer ejercicio & 1,21 & 0,46 & 111 & 81,6 & 22 & 16,2 & 3 & 2,2 \\
\hline 20. Me gusta la competición & 1,24 & 0,52 & 109 & 80,1 & 21 & 15,4 & 6 & 4,4 \\
\hline 22. Me gusta practicar deporte & 1,16 & 0,46 & 119 & 87,5 & 12 & 8,8 & 5 & 3,7 \\
\hline 23. Quiero mejorar mi nivel & 1,17 & 0,43 & 116 & 85,3 & 17 & 12,5 & 3 & 2,2 \\
\hline 24. Quiero estar físicamente bien & 1,24 & 0,48 & 106 & 77,9 & 27 & 19,9 & 3 & 2,2 \\
\hline 29. Me gusta divertirme & 1,26 & 0,51 & 104 & 76,5 & 28 & 20,6 & 4 & 2,9 \\
\hline \multicolumn{9}{|l|}{ Social } \\
\hline 02. Quiero estar con mis amigos & 1,65 & 0,68 & 64 & 47,1 & 56 & 41,2 & 16 & 11,8 \\
\hline 05. Me gusta viajar & 1,65 & 0,68 & 63 & 46,3 & 56 & 41,2 & 16 & 11,8 \\
\hline 08. Me gusta el trabajo en equipo & 1,49 & 0,64 & 80 & 58,8 & 45 & 33,1 & 11 & 8,1 \\
\hline 11. Me gusta hacer nuevos amigos & 1,57 & 0,63 & 69 & 50,7 & 57 & 41,9 & 10 & 7,4 \\
\hline 16. Me gusta tener algo que hacer & 1,60 & 0,61 & 64 & 47,1 & 63 & 46,3 & 9 & 6,6 \\
\hline 18. Me gusta el espíritu de equipo & 1,51 & 0,66 & 79 & 58,1 & 45 & 33,1 & 12 & 8,8 \\
\hline 19. Me gusta salir de casa & 1,78 & 0,71 & 52 & 38,2 & 62 & 45,6 & 22 & 16,2 \\
\hline $\begin{array}{l}\text { 30. Me gusta utilizar el equipo o } \\
\text { instalaciones }\end{array}$ & 1,60 & 0,66 & 68 & 50,0 & 55 & 40,4 & 13 & 9,6 \\
\hline \multicolumn{9}{|l|}{ Auto estima } \\
\hline 09. Es por satisfacer a mis padres & 2,39 & 0,77 & 24 & 17,6 & 35 & 25,7 & 76 & 55,9 \\
\hline $\begin{array}{l}\text { 12. Me gusta hacer algo en lo que } \\
\text { destaco }\end{array}$ & 1,51 & 0,60 & 73 & 53,7 & 56 & 41,2 & 7 & 5,1 \\
\hline 14. Me gustan las recompensas & 1,66 & 0,69 & 63 & 46,3 & 55 & 40,4 & 17 & 12,5 \\
\hline 21. Me gusta sentirme importante & 1,87 & 0,74 & 47 & 34,6 & 60 & 44,1 & 29 & 21,3 \\
\hline 25. Quiero ser popular & 2,35 & 0,74 & 21 & 15,4 & 45 & 33,1 & 68 & 50,0 \\
\hline 27. Por influencia de los entrenadores & 2,29 & 0,73 & 22 & 16,2 & 52 & 38,2 & 62 & 45,6 \\
\hline
\end{tabular}




\begin{tabular}{lcccccccc} 
28. Quiero alcanzar un status-élite & 1,65 & 0,73 & 68 & 50,0 & 48 & 35,3 & 20 & 14,7 \\
\hline Competencia & & & & & & & & \\
07. Me gustan las emociones & 1,42 & 0,57 & 72 & 52,9 & 54 & 39,7 & 10 & 7,4 \\
17. Me gusta la acción & 1,46 & 0,62 & 83 & 61,0 & 44 & 32,4 & 9 & 6,6 \\
26. Me gustan los desafíos & 1,35 & 0,57 & 94 & 69,1 & 36 & 26,5 & 6 & 4,4 \\
\hline
\end{tabular}

M - media; DT - desviación típica; f - frecuencia; \% - porcentaje.

\section{Análisis comparativo entre grupos}

Las tablas6 indica la comparación entre grupos, entre sexo y los cuatro factores, y entre discapacidad y los cuatro factores, respectivamente, indicando los valores medios, desviaciones típicas y pruebas $t$ de student. Como se puede verificar, hay similitud de resultados obtenidos en la escala, sin que haya diferencia significativa entre hombres y mujeres, ni entre ciegos-discapacitados visuales y discapacitados físicos.

Tabla 6. Comparación entre Factores y Sexo, y Factores y Discapacidad

Competencia Autoestima $\quad$ Social $\quad \begin{gathered}\text { Condición Física } \\ \text { / Salud }\end{gathered}$

\begin{tabular}{|c|c|c|c|c|c|c|c|c|}
\hline Sexo & Hombre & Mujer & Hombre & Mujer & Hombre & Mujer & Hombre & Mujer \\
\hline $\mathrm{N}$ & 106 & 30 & 103 & 29 & 105 & 30 & 106 & 30 \\
\hline Media & 4,28 & 4,03 & 13,90 & 13,38 & 12,95 & 12,33 & 11,18 & 10,93 \\
\hline Desv. Típ. & 1,42 & 1,40 & 3,05 & 3,57 & 3,51 & 3,69 & 3,23 & 1,91 \\
\hline prueba $t$ & \multicolumn{2}{|c|}{0,859} & \multicolumn{2}{|c|}{0,719} & \multicolumn{2}{|c|}{0,819} & \multicolumn{2}{|c|}{0,524} \\
\hline Significancia & \multicolumn{2}{|c|}{0,395} & \multicolumn{2}{|c|}{0,476} & \multicolumn{2}{|c|}{0,417} & \multicolumn{2}{|c|}{0,602} \\
\hline & \multicolumn{2}{|c|}{ Competencia } & \multicolumn{2}{|c|}{ Autoestima } & \multicolumn{2}{|c|}{ Social } & \multicolumn{2}{|c|}{$\begin{array}{c}\text { Condición Física } \\
\text { / Salud }\end{array}$} \\
\hline Discapacidad & C.DV & $\mathrm{FM}$ & C.DV & FM & C.DV & FM & C.DV & FM \\
\hline $\mathrm{N}$ & 55 & 79 & 55 & 75 & 55 & 78 & 55 & 79 \\
\hline Media & 4,44 & 4,06 & 14,36 & 13,43 & 13,15 & 12,62 & 11,56 & 10,82 \\
\hline Desv. Típ. & 1,48 & 1,36 & 2,66 & 3,47 & 3,66 & 3,51 & 3,56 & 2,53 \\
\hline prueba $t$ & \multicolumn{2}{|c|}{1,486} & \multicolumn{2}{|c|}{1,675} & \multicolumn{2}{|c|}{0,836} & \multicolumn{2}{|c|}{1,327} \\
\hline Significancia & \multicolumn{2}{|c|}{0,140} & \multicolumn{2}{|c|}{0,096} & \multicolumn{2}{|c|}{0,405} & \multicolumn{2}{|c|}{0,188} \\
\hline
\end{tabular}

C.DV - ciegos-discapacitados visuales; FM - discapacitados físicos 


\section{DISCUSION Y CONCLUSIONES}

Tradicionalmente se ha venido utilizando el deporte adaptado como procedimiento para la rehabilitación de las personas con discapacidad, si bien recientemente se le está prestando mayor atención como elemento propio del desarrollo integral de la persona, atribuyendo al deporte los mismos beneficios para los deportistas con discapacidad que sin ella, ya que se considera que desempeña una importante función en el desarrollo físico, psicológico y social de quien lo practica. Es por ello que el objetivo de esta investigación se ha basado en analizar las motivaciones para la práctica deportiva de las personas con discapacidad, recogiendo una muestra amplia de deportistas federados en diversos deportes.

La importancia concedida por todos los atletas discapacitados a los diferentes ítems de la escala nos indica que los motivos más importantes son"practicar deporte", "mejorar el nivel", "hacer ejercicio", "competir", "estar físicamente bien", "divertirme", "mantenerse en forma", "ganar"y "mejorar las habilidades". Estos resultados van en línea con lo encontrado por Gill y cols. (1983), Gould y cols. (1985), García Ferrando (1993), Balaguer y Atienza (1994) y Villamarín, Mauri, y Sanz (1998), lo que demuestra que los jóvenes tienen motivos similares para practicar diferentes deportes. En el último estudio del Centro de Investigaciones Sociológicas (CIS) y el Consejo Superior de Deportes, del año 2010, (Garcia Ferrando, y Llopis Goig. 2010) indica que el motivo más citado para hacer deporte fue por hacer ejercicio físico, pues así lo señala el $70 \%$ de las personas que hacen deporte, de acuerdo con los datos que nosotros hemos obtenido, más del $80 \%$ desean hacer ejercicio físico. El estudio del CIS indica que el segundo motivo, con el 50\%, es practicar deporte con carácter recreativo. Estos datos están muy de acuerdo con los obtenidos en nuestro trabajo, indicando que la principal motivación de la práctica deportiva, el $76,5 \%$, es por diversión, tal como se indica en la tabla 2.

No son tan representativos los datos de la encuesta del CIS con motivo de mejora y mantenimiento de la salud, reflejan un $48 \%$ como tercer motivo más citado, y en cambio en los deportistas discapacitados hemos encontrado un valor más alto de motivación, un 74,3\%.

En el otro extremo, encontramos que los motivos que fueron valorados por unos y por otros como menos importantes fueron: "satisfacer a los padres", "ser importante y popular" "sentirse importante" y "por influencia de los entrenadores". Este último ítem reafirma lo encontrado por Gould y cols. (1985), que recogieron que el entrenador pierde influencia en la motivación de la práctica deportiva en función de la edad de los practicantes, por lo que lleva a pensar en la gran importancia que tiene el entrenador en las etapas de iniciación deportiva como elemento motivador. En el estudio del CIS y CSD los valores menos citados en su encuesta fueron "porque le gusta competir" con 4,8\%, "por escapar de lo habitual" $15,1 \%$ y por mantener la línea $22,3 \%$.

No obstante debemos tener en cuenta que el estudio del CIS y CSD se realizó con toda la población española y el nuestro fue realizado con deportistas de 
caracter federado, lo que puede indicar que hayan algunas diferencias notables, como la que obtuvimos en la motivación respecto a la competición que nos dio un $4,8 \%$ por el $80,1 \%$ en el deporte adaptado federado.

\section{REFERENCIAS}

Balaguer, I. y Atineza, F. (1994). Principales motivos de los jóvenes para jugar al tenis. Apunts de Educación Física y Deportiva, 31, 285-299.

Cecchini, J.A., Echevarría, L.M., y Méndez, A. (2003). Intensidad de la motivación hacia el deporte en edad escolar. Oviedo. Universidad deOviedo

Cecchini, J.A., Méndez, A. y Muñiz, J. (2002). Motives for practicing sport in Spanish Schoolchildren. Psicothema, 14 (3), 523-531.

Cei, A., Buonamano, R. y Mussino, A. (1993). Participation motivation in Italian youth sport: A preliminary study. FEPSAC Congress on Emotions in Sport, 22-25.

Comité Paralímpico Español. (2006). Paralímpicos.Madrid: Autor.

Durand, M. (1988). El niño y el deporte. Madrid: Editorial Paidós.

García Ferrando, M. (1993). Tiempo libe y actividades deportivas en la juventud en España. Madrid: Ministerio de Asuntos Sociales.

Garcia Ferrando, M. y Llopis Goig, R. (2010) Ideal democrático y bienestar personal: Encuesta sobre los hábitos deportivos en España.Centro de Investigaciones Sociológicas y Consejo Superior de Deportes.

Gill, D.L., Gross, J.B. y Huddleton, S. (1983). Participation motivation in youth sports. International Journal of Sports Psychology, 1, 1-14.

Gould, D., Feltz, D. y Weiss, M. (1985). Motives for participating in competitive swimming. International Journal of Sports Psychology 16, 126-140.

Guthrie, S.R. (1999). Managing imperfection in a perfectionistic culture: Physical activity and disability management among women with disabilities. Quest, 51, 369-381.

Guthrie, S.R. y Castelnuovo, S. (2001). Disability management among women with physical impairments: The contribution of physical activity. Sociology of Sport Journal, 18, 5-20.

Gutiérrez, M. y Caus, N. (2006). Análisis de los motivos para la Participación en actividades físicas de personas con y sin discapacidad. Revista Internacional de Ciencias del Deporte. 2 (2), 49-64. http://www.cafyd.com/REVISTA/art4n2a06.pdf

Klint, K.A. y Weiss M.R. (1987). Perceived competence and motives forparticipating in youth sport: A test of Harter's competence motivation theory. Journal of Sport Psychology, 9, 55-65.

Lazaro, I.; Villamarin, F. y Limonero, J. (1996). Motivación para participar y auto-eficacia en jóvenes jugadores de baloncesto. En Pérez, E. y Caracuel, J.C. (edit.), Actas del IV Congreso Nacional y del IV Congreso Andaluz de Psicología del Deporte (pp.207-215). Instituto Andaluz de Deporte/Junta de Andalucía: Málaga. 
Martin, J.J. (1999). Predictors of social physique anxiety in adolescent swimmers withphysical disabilities. Adapted Physical Activity Quarterly, 16, 75-85.

Nunnally, J. C., y Bernstein, I. J. (1995). Teoría psicométrica (3ra. ed.). México, D. F: physical impairments: The contribution of physical activity. Sociology of Sport.

OMS (1980). Clasificación Internacional de Deficiencias, Discapacidades y Minusvalías. Ministerio de Trabajo y Asuntos Sociales. Madrid.

Ryckman, R. y Hamel, J. (1995). Male and female adolescents' motives related to involvement in organized team sports. International Journal of Sports Psychology, 26, 383-397.

Villamarin, F., Mauri, C. y Sanz, A. (1998). Competencia percibida y motivación durante la iniciación en la práctica del tenis. Revista de Psicología del Deporte, 13, 41-56.

Wang, J. y Wiese-Bjornstal, D.M. (1996). The relationship of school type and gender to motives for sport participation among youth in the People's Republic of China. International Journal of Sports Psychology, 28, 13-24. 